PROCEEDINGS OF THE

AMERICAN MATHEMATICAL SOCIETY

Volume 126, Number 12, December 1998, Pages 3727-3731

S $0002-9939(98) 04560-2$

\title{
THE ORDER OF A MERIDIAN OF A KNOTTED KLEIN BOTTLE
}

\author{
KATSUYUKI YOSHIKAWA
}

(Communicated by Dale Alspach)

\begin{abstract}
We consider the order of a meridian (of the group) of a Klein bottle smoothly embedded in the 4 -sphere $S^{4}$. The order of a meridian of a Klein bottle in $S^{4}$ is a non-negative even integer. Conversely, we prove that, for every non-negative even integer $n$, there exists a Klein bottle in $S^{4}$ whose meridian has order $n$.
\end{abstract}

\section{INTRODUCTION}

Let $F$ be a Klein bottle smoothly embedded in the 4 -sphere $S^{4}$. Let $N(F)$ be the tubular neighborhood of $F$ in $S^{4}$. Then $N(F)$ is a $D^{2}$-bundle over $F$ and the boundary $\partial N(F)$ of $N(F)$ is an $S^{1}$-bundle over $F$. A fiber $S^{1} \times\{*\}$, where $* \in F$, is called a meridian of $F$. Let $G$ be the group of $F$, i.e., $\pi_{1}\left(S^{4}-F\right)$. An element of $G$ represented by a meridian of $F$ is also called a meridian of $G$ (or $F$ ). We consider the order of a meridian (of the group) of a Klein bottle $F$ in $S^{4}$. Since $H_{1}(G) \cong Z_{2}$, the order of a meridian of a Klein bottle $F$ in $S^{4}$ is a non-negative even integer. For example, the connected sum of any 2-knot and a standard Klein bottle in $S^{4}$ has a meridian of order 2 . In this paper, more generally, we prove that

Theorem. For every non-negative even integer $n$, there exists a Klein bottle in $S^{4}$ whose meridian has order $n$.

\section{Preliminaries}

Let $K+h$ denote the Klein bottle in $S^{4}$ obtained by attaching a non-orientable 1-handle $h$ to a 2 -knot $K$ in $S^{4}$. We may assume that the non-orientable 1-handle $h$ on $K$ is attached very near the base point of the group $G\left(=\pi_{1}\left(S^{4}-K\right)\right)$ of $K$. Then we can consider that the core of $h$ represents an element $g(h)$ of $G$. Conversely, given an element $g$ of $G$, there exists a non-orientable 1-handle $h$ on $K$ such that $g=g(h)$. For a subset $S$ of a group $H$, we denote by $\langle\langle S\rangle\rangle$ the normal closure of $S$ in $H$.

Proposition 1. Let $K$ be a 2-knot in $S^{4}$ and $h$ a non-orientable 1-handle on $K$. Then the group of the Klein bottle $K+h$ in $S^{4}$ is given by $\pi_{1}\left(S^{4}-K\right) /$ $\left\langle\left\langle x g(h) x g(h)^{-1}\right\rangle\right\rangle$, where $x$ is a meridian of $K$.

Proof. In the same way as in Lemma 9 of [1], we can prove the proposition.

Received by the editors April 9, 1997.

1991 Mathematics Subject Classification. Primary 57Q45.

Key words and phrases. Klein bottle, meridian. 
The following is immediately obtained from Proposition 1.

Lemma 2. Let $G_{0}$ be a 2-knot group and $x$ a meridian of $G_{0}$. Then, for any element $g$ of $G_{0}$, the quotient group $G_{0} /\left\langle\left\langle x g x g^{-1}\right\rangle\right\rangle$ is the group of some Klein bottle in $S^{4}$.

Lemma 3. Let $n$ be a non-negative even integer. Let $H$ be a finitely generated group satisfying the following:

(1) There exists an element $h$ of $H$ such that $\langle\langle h\rangle\rangle=H$.

(2) There exists an element $u$ of $H$ such that $u h u^{-1}=h^{-1}$.

(3) $h^{\frac{n}{2}} \in C(H)$, where $C(H)$ is the center of $H$.

(4) $|h|=n$, where $|h|$ denotes the order of $h$.

Then there exists a Klein bottle in $S^{4}$ whose meridian has order $n$.

Proof. Let $m=\frac{n}{2}$. By (1), there exist some 1-knot $k$ and an epimorphism $\phi$ of $\pi_{1}\left(S^{3}-k\right)$ onto $H$ such that $\phi(x)=h$, where $x$ is a meridian of $k$ [2]. Let $K$ be the $m$-twist-spun 2-knot of the 1-knot $k$. Then the group $\pi_{1}\left(S^{4}-K\right)$ of $K$ is given by $\pi_{1}\left(S^{3}-k\right) /\left[x^{m}, \pi_{1}\left(S^{3}-k\right)\right]$. Therefore, by $(3), \phi$ induces the epimorphism $\phi_{*}$ of $\pi_{1}\left(S^{4}-K\right)$ onto $H$ such that $\phi_{*} \alpha=\phi$, where $\alpha: \pi_{1}\left(S^{3}-k\right) \rightarrow \pi_{1}\left(S^{4}-K\right)$ is the natural homomorphism. Then $\alpha(x)$ is a meridian of $K$. Let $G=\pi_{1}\left(S^{4}-K\right) /$ $\left\langle\left\langle\alpha(x) \tilde{u} \alpha(x) \tilde{u}^{-1}\right\rangle\right\rangle$, where $\tilde{u} \in \phi_{*}^{-1}(u)$, and let $\beta: \pi_{1}\left(S^{4}-K\right) \rightarrow G$ be the natural homomorphism. Then, by Lemma $2, G$ is the group of some Klein bottle in $S^{4}$ and $\beta \alpha(x)$ is a meridian of $G$. We show that $\beta \alpha(x)$ has order $n$ in $G$. Since $\beta \alpha(x)^{m} \in C(G)$, we have

$$
\beta \alpha(x)^{m}=\beta(\tilde{u}) \beta \alpha(x)^{m} \beta(\tilde{u})^{-1}=\beta \alpha(x)^{-m} .
$$

Therefore we see that $\beta \alpha(x)^{2 m}=1$. On the other hand, by $(2), \phi_{*}$ induces the epimorphism $\psi$ of $G$ onto $H$ such that $\psi \beta \alpha=\phi_{*} \alpha=\phi$. Thus, since $\psi \beta \alpha(x)=$ $\phi(x)=h$, it follows from (4) that $|\beta \alpha(x)|=|h|=2 m=n$. The proof is completed.

Lemma 4. Let $n$ be a positive even integer. Let $H_{*}$ be a finitely generated group satisfying the following:

(a) There exists an element $h_{*}$ of $H_{*}$ such that $\left\langle\left\langle h_{*}\right\rangle\right\rangle=H_{*}$.

(b) There exists an element $u_{*}$ of $H_{*}$ such that $u_{*} h_{*} u_{*}^{-1}=h_{*}^{-1}$.

(c) $h_{*}^{\frac{n}{2}} \in C\left(H_{*}\right)$.

(d) $\left|h_{*}\right|=n$.

Then, for any positive integer $m$ such that $(m, n)=1$, there exists a Klein bottle in $S^{4}$ whose meridian has order $m n$.

Proof. Let $H=H_{*} \times A_{2 m}$, where $A_{2 m}$ is the alternating group of degree $2 m$. (Since $m$ is odd, we have $2 m=2$ or $>5$. Therefore $A_{2 m}$ is simple.) We will show that $H$ satisfies the conditions (1)-(4) of Lemma 3.

(1) Let $h=h_{*} b$, where $b=(1,2, \ldots, m)(m+1, m+2, \ldots, 2 m) \in A_{2 m}$. Then we have $|b|=m$ and $\left(\left|h_{*}\right|,|b|\right)=1$. Therefore, by $(\mathrm{a})$, we see that

$$
H /\langle\langle h\rangle\rangle=H_{*} \times A_{2 m} /\left\langle\left\langle h_{*}, b\right\rangle\right\rangle=A_{2 m} /\langle\langle b\rangle\rangle \cong 1 .
$$

(2) Let $c=\{(1, m)(2, m-1) \cdots(k, k+2)\} \cdot\{(m+1,2 m)(m+2,2 m-1) \cdots$ $(3 k+1,3 k+3)\} \in A_{2 m}$, where $k=(m-1) / 2$. Then we have $c b c^{-1}=b^{-1}$. Let $u=u_{*} c$. Then, by (b), we get

$$
u h u^{-1}=\left(u_{*} c\right)\left(h_{*} b\right)\left(c^{-1} u_{*}^{-1}\right)=\left(u_{*} h_{*} u_{*}^{-1}\right)\left(c b c^{-1}\right)=h_{*}^{-1} b^{-1}=b^{-1} h_{*}^{-1}=h^{-1} .
$$


(3) By (c), we have

$$
h^{m n / 2}=\left(h_{*} b\right)^{m n / 2}=\left(h_{*}^{n / 2}\right)^{m}\left(b^{n / 2}\right)^{m}=\left(h_{*}^{n / 2}\right)^{m} \in C\left(H_{*}\right) \subset C(H) .
$$

(4) By (d), we get $|h|=\left|h_{*}\right| \cdot|b|=m n$.

Therefore, by Lemma 3, we complete the proof.

The following corollary immediately follows from Lemma 4.

Corollary 5. Let $n$ be a positive even integer. If there exists a Klein bottle $F$ in $S^{4}$ such that $x^{\frac{n}{2}} \in C(G)$ and $|x|=n$, where $x$ is a meridian of the group $G$ of $F$, then, for any positive integer $m$ such that $(m, n)=1$, there exists a Klein bottle in $S^{4}$ whose meridian has order $m n$.

\section{Proof of the Theorem}

Case (1): $n=0$.

Let

$$
G_{1}=\left\langle a, b, z: a=b^{-1} a^{-1} b a b, z a z^{-1}=b^{-1}, z b z^{-1}=a b^{-1} a^{-1}\right\rangle
$$

and $A=\left\langle a, b: a=b^{-1} a^{-1} b a b\right\rangle$. The mapping of $A$ to itself given by $a \rightarrow b^{-1}$ and $b \rightarrow a b^{-1} a^{-1}$ induces the automorphism of $A$. Therefore $G_{1}$ is the extension of $A$ by the infinite cyclic group $\langle z:\rangle$. Since $A$ is the group of the trefoil 1-knot, $A$ is torsion-free and so is $G_{1}$. Let $y=a b a^{-1} b^{-1}$. Then $y$ commutes with $z$. Therefore the subgroup $B_{1}$ of $G_{1}$ generated by $y$ and $z$ is free abelian of rank 2 . Let $G_{2}$ be the group of a non-trivial 1-knot and $B_{2}$ the peripheral subgroup of $G_{2}$. Let $H$ be the free product of $G_{1}$ and $G_{2}$ with the subgroups $B_{1}$ and $B_{2}$ amalgamated under the isomorphism of $B_{1}$ on $B_{2}$ given by $y \rightarrow c$ and $z \rightarrow l$, where $c$ and $l$ are a meridian and a longitude of $G_{2}$. Then, if $h=b$ and $u=z a$ in Lemma 3, we can see that $H$ satisfies the conditions (1)-(4) of Lemma 3 . Therefore there exists a Klein bottle in $S^{4}$ whose meridian has order 0 .

Case (2): $n=2(2 k+1)$ or $4(2 k+1), k \geq 0$.

First, when $n=2(2 k+1)$, let $H_{*}=Z_{2}$. Then, since the group of a standard Klein bottle in $S^{4}$ is $Z_{2}$, the assertion follows from Corollary 5 . Next, when $n=4(2 k+1)$, let

$$
H_{*}=\left\langle a, b, x_{*}: a^{2}=b^{3}=\left(b^{-1} a\right)^{3}, x_{*} a x_{*}^{-1}=a^{-1}, x_{*} b x_{*}^{-1}=b^{-1}, x_{*}^{2}=a^{2}\right\rangle .
$$

Then $H_{*}$ is the extension of $T_{*}$ by the cyclic group $Z_{2}$ of order 2 , where $T_{*}$ is the binary tetrahedral group presented by $\left\langle a, b: a^{2}=b^{3}=\left(b^{-1} a\right)^{3}\right\rangle$. Then we can show that $\left\langle\left\langle x_{*}\right\rangle\right\rangle=H_{*}, a x_{*} a^{-1}=x_{*}^{-1}, x_{*}^{2} \in C\left(H_{*}\right)$ and $\left|x_{*}\right|=|a|=4$. Let $h_{*}=x_{*}$ and $u_{*}=a$ in Lemma 4 . Then we can see that $H_{*}$ satisfies the conditions (a)-(d) of Lemma 4. Therefore we complete the proof of case (2).

Case (3): $n=8 m, m>0$.

Let

$$
G=\left\langle x, y: y=x^{m} y^{m} x y^{-m} x^{-m}, x^{2 m} y x^{-2 m}=y^{-1}\right\rangle .
$$

Then, by the second relation, we see that

$$
x^{4 m} y x^{-4 m}=x^{2 m} y^{-1} x^{-2 m}=y .
$$

Hence $y^{4 m}=x^{m} y^{m} x^{4 m} y^{-m} x^{-m}=x^{4 m}$. Therefore we have

$$
y^{-4 m}=x^{2 m} y^{4 m} x^{-2 m}=x^{2 m} x^{4 m} x^{-2 m}=x^{4 m}=y^{4 m} \text {. }
$$


It follows that $y^{8 m}=1$. Let $\phi: G \rightarrow S L(2, C)$ be the homomorphism defined by $\phi(x)=a$ and $\phi(y)=w a w^{-1}$, where

$$
a=\left(\begin{array}{cc}
e^{\pi i / 4 m} & 0 \\
0 & e^{-\pi i / 4 m}
\end{array}\right) \quad \text { and } \quad w=\left(\begin{array}{cc}
\frac{1}{2}(i+1) & \frac{1}{2}(1-i) \\
-\frac{1}{2}(i+1) & \frac{1}{2}(1-i)
\end{array}\right) .
$$

Since $|a|=8 m$, we see that $|x|=|y|=8 m$.

Let $G_{0}=\left\langle x, y: y=x^{m} y^{m} x y^{-m} x^{-m}\right\rangle$. Then $G_{0}$ is the group of a ribbon 2-knot. Therefore, by Lemma 2, $G$ is the group of some Klein bottle in $S^{4}$.

Thus we complete the proof of the theorem.

\section{REMARKS}

(1) The group $G_{0}$ of the 2-twist-spun 2-knot of the (-2,3,3)-pretzel 1-knot is presented by

$$
\left\langle a, b, x_{*}: a^{2}=b^{3}=\left(b^{-1} a\right)^{3}, x_{*} a x_{*}^{-1}=a^{-1}, x_{*} b x_{*}^{-1}=b^{-1}\right\rangle .
$$

Since $G_{0} /\left\langle\left\langle x_{*} a x_{*} a^{-1}\right\rangle\right\rangle$ is isomorphic to the group $H_{*}$ given by $\left(^{*}\right)$, it follows from Lemma 2 that $H_{*}$ is the group of some Klein bottle in $S^{4}$.

(2) The group $G$ of a Klein bottle in $S^{4}$ has deficiency less than one because $H_{1}(G) \cong Z_{2}$. For each non-negative even integer $n$, the problem arises whether there exists a Klein bottle in $S^{4}$ whose group has a meridian of order $n$ and has deficiency zero. From the proofs of cases (1) and (3) in Section 3, we can see that, if $n=8 m(m \geq 0)$, then there exists such a Klein bottle in $S^{4}$. (In case of $n=0$, for instance, the group presented by

$$
\begin{aligned}
& \left\langle a, b, c, d, z: a=b^{-1} a^{-1} b a b, z b z^{-1}=a b^{-1} a^{-1},\right. \\
& \left.d=c^{-1} d^{-1} c d c, a b a^{-1} b^{-1}=c, z=c^{4} d^{-1} c^{-2} d^{-1}\right\rangle
\end{aligned}
$$

is such an example because $\left\langle a, b, c, d, z: a=b^{-1} a^{-1} b a b, d=c^{-1} d^{-1} c d c, a b a^{-1} b^{-1}=\right.$ $\left.c, z=c^{4} d^{-1} c^{-2} d^{-1}\right\rangle$ is the group of a ribbon 2-knot.) The case $n \neq 8 m(m \geq 0)$ is still open.

(3) It is known that a meridian of a projective plane smoothly embedded in $S^{4}$ has order 2 or 4 (cf. [4, $\S \mathrm{VI}]$ ). In case of order 2, there exists such a projective plane in $S^{4}$ (e.g., the connected sum of any 2-knot and a standard projective plane in $\left.S^{4}([3],[4])\right)$. It remains open whether or not there exists a projective plane in $S^{4}$ whose meridian has order 4 .

(4) Using the Theorem, we can easily prove that, for every non-negative even integer $n$, there exists a non-orientable surface of (non-orientable) genus $2 m(m>0)$ in $S^{4}$ whose meridian has order $n$.

\section{REFERENCES}

[1] J. Boyle, Classifying 1-handles attached to knotted surfaces, Trans. Amer. Math. Soc. 306 (1988), 475-487. MR 89f:57032

[2] F. González-Acuña, Homomorphisms of knot groups, Ann. of Math. 102 (1975), 373-377. MR 52:576 
[3] S. Kinoshita, On the Alexander polynomial of 2-spheres in a 4-sphere, Ann. of Math. 74 (1961), 518-531. MR 24:A2960

[4] T. M. Price and D. M. Roseman, Embeddings of the projective plane in four space, preprint.

Faculty of Science, Kwansei Gakuin University, Uegahara Nishinomiya, Hyogo 662 8501, JAPAN

E-mail address: yoshikawa@kgupyr.kwansei.ac.jp 